\title{
Comparative Study of Diagnostic Markers in Neonatal Sepsis
}

\author{
Sonawane VB ${ }^{1}$, Gaikwad SU², Kadam NN³ , Gavhane $\mathrm{J}^{4}$
}

\begin{abstract}
Introduction: Neonatal septicemia is one of the commonest causes of neonatal mortality and morbidity. The objectives of this study were intended for qualitative and quantitative analysis of diagnostic markers in neonatal sepsis. Materials and Methods: This is a hospital based study conducted over three years (Aug 2005 - Aug 2008). Hundred and sixty neonates, delivered in the hospital, having risk factors for neonatal sepsis, along with those coming to hospital with signs and symptoms of sepsis up to 28 days of life (as study group) along with normal newborns admitted to the postnatal ward without high risk factors (control group) were enrolled for this study. Comparative study on various diagnostics markers such blood culture, CBC, CRP, IT ratio and Micro-ESR was carried out to know their sensitivity and specificity. Results: E.Coli was the most common organism responsible for sepsis. CRP was reported to be highly sensitive (84.21\%), and CBC was highly specific (75.00\%), IT ratio has sensitivity of $62.5 \%$ and specificity of $56.25 \%$ while MicroESR has shown sensitivity of $50.0 \%$ and specificity of $62.5 \%$, Out of 160 cases, blood culture (BacTalert) showed growth in 48 cases in study group while two cases in control group. Thus blood culture positivity was $60 \%$. Conclusion: Blood culture is the gold standard for the diagnosis of septicemia. CRP is most sensitive while $\mathrm{CBC}$ is most specific marker in neonatal sepsis.
\end{abstract}

Key words: Neonatal Sepsis, Diagnostic Markers, Mortality

\section{Introduction}

$\mathrm{N}$ eonatal Septicemia is the leading cause neonatal mortality and morbidity in India. It is estimated that $20 \%$ of all neonates develop sepsis ${ }^{1}$ and is responsible for $30-50 \%$ of total neonatal death in developing countries ${ }^{2}$. Accurate and timely diagnosis of neonatal sepsis still remains a major challenge to the pediatricians and neonatologists. Mortality due to neonatal sepsis is preventable and if diagnosed early the outcome is better. Several indicators have been evaluated as "septic screen" for the early diagnosis of neonatal sepsis like CBC, CRP, Micro-ESR, IT ratio, and Blood Culture. There is a constant search for better and ideal diagnostic marker. Ideal marker should be sensitive, specific and easily available. Its results should be immediate and reproducible.
'Dr. Vijay B. Sonawane, MBBS, MD, Assistant Professor, ${ }^{2}$ Dr. Sonali U Gaikwad, MBBS, MD, Senior Resident, ${ }^{3}$ Dr. Nitin N Kadam, MBBS, MD, Professor and Head of Department, ${ }^{4}$ Dr. Jitendra Gavhane, MBBS, MD, Assistant Professor. All from the Department of Paediatrics, MGM Medical College and Hospital, Navi Mumbai, Maharashtra, India.

\section{Address for correspondence:}

Dr. Vijay Baburao Sonawane, Assistant Professor, Department of Paediatrics,

MGM Medical College and Hospital,

Navi Mumbai, Maharastra, India.

E-mail: vijay_Itm@yahoo.co.in

\section{How to cite}

Sonawane VB, Gaikwad SU, Kadam NN, Gavhane J. Comparative Study of Diagnostic Markers in Neonatal Sepsis. J Nepal Paediatr Soc 2014;34(2):111-114.

doi: http://dx.doi.org/10.3126/jnps.v34i2.9788

This work is licensed under a Creative Commons Attribution 3.0 License.

\section{(c) (i)}

\section{Materials and Methods}

In our study involved 160 babies delivered in the hospital, having risk factors, along with those coming to hospital with signs and symptoms of sepsis up to 28 days of life (Study group) also normal newborns admitted to the postnatal ward without high risk factors (Control group). Newborns were treated with antibiotics for clinical evidence of sepsis \& positive septic score as per neonatal sepsis score system (Table 1). 
Table 1: Neonatal sepsis score system ${ }^{3}$

\begin{tabular}{|l|l|}
\hline Score 1 & $\begin{array}{l}\text { Maternal fever in } 3^{\text {rd }} \text { trimester, instrumental } \\
\text { delivery, intubations, exchange transfusion, } \\
\text { LBW, outside delivery, abdominal distention, } \\
\text { irritability, lethargy, convulsion, apnea }\end{array}$ \\
\hline Score 2 & $\begin{array}{l}\text { Hypothermia, fever, local infection, refusal } \\
\text { to feed, feed Intolerance, vomiting, loose } \\
\text { stools, meconium aspiration }\end{array}$ \\
\hline Score 3 & $\begin{array}{l}\text { LPV >12 hrs, chorioamnionitis, cord } \\
\text { erythema, foul smelling (cord) }\end{array}$ \\
\hline Score 4 & Sclerema, meningitis, DIC, NEC \\
\hline
\end{tabular}

Score1=risk of infection, Score2=need septic work up to exclude, Score 3 or more=investigate and treat

A detailed history was taken and examination was done. Following laboratory tests were done as soon as presumptive diagnosis of sepsis was made based on septic score system and on clinical grounds. All investigations were done within 24 hours of birth or at presentation before starting antibiotics like $\mathrm{CBC}$, CRP with titer, Micro ESR, Immature to Total (IT) ratio, peripheral smear for toxic granules and band forms, blood culture (Bac Talert), x-ray chest, CSF whenever indicated etc. Soon after birth, $1 \mathrm{ml}$ of venous blood was drawn for blood culture. Also $5 \mathrm{ml}$ of venous blood was collected for TLC, DLC, peripheral blood smear, micro-ESR and CRP. CRP was sent at 12 hours of life in newborns with high risk factors for sepsis.

This study was approved by Ethical Committee of this hospital. Informed Written Consent was obtained from parents before entry into this study. Data was collected, classified, tabulated and analyzed. Tests of significance were applied at appropriate places and interpretation was done accordingly. To evaluate the difference between the categories, McNemar Chi Square test was used as a test of significance. A $p$-value of less than 0.05 was considered statistically significant.

\section{Results}

Among the babies of study group, 48 (60\%) were blood culture (BacTalert) positive while in control group, only 2 (2.5\%) were blood culture (BacTalert) positive (Fig.1).

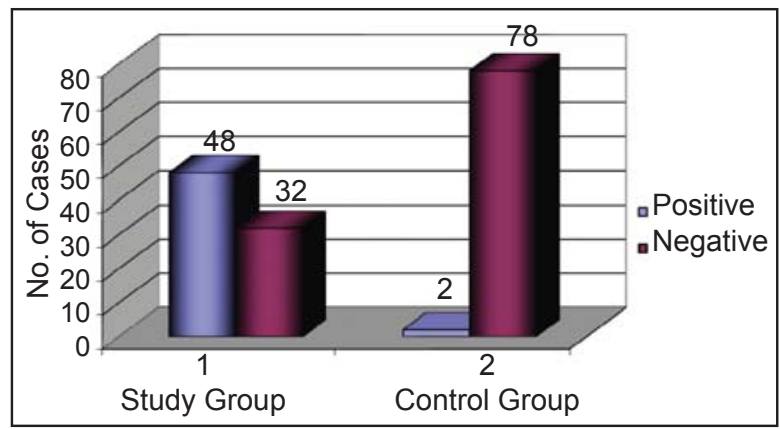

Fig 1: Distribution of Cases According to Blood Culture

In study group, E-coli comprised the maximum number of cases accounting for sepsis i.e. 7 (17.5\%) while there were no growths in $16(40 \%)$ cases. In control group, only 1 case $(2.5 \%)$ showed growth of Acinetobacter baumanii and 39 cases (97.5\%) were sterile (Fig. 2).

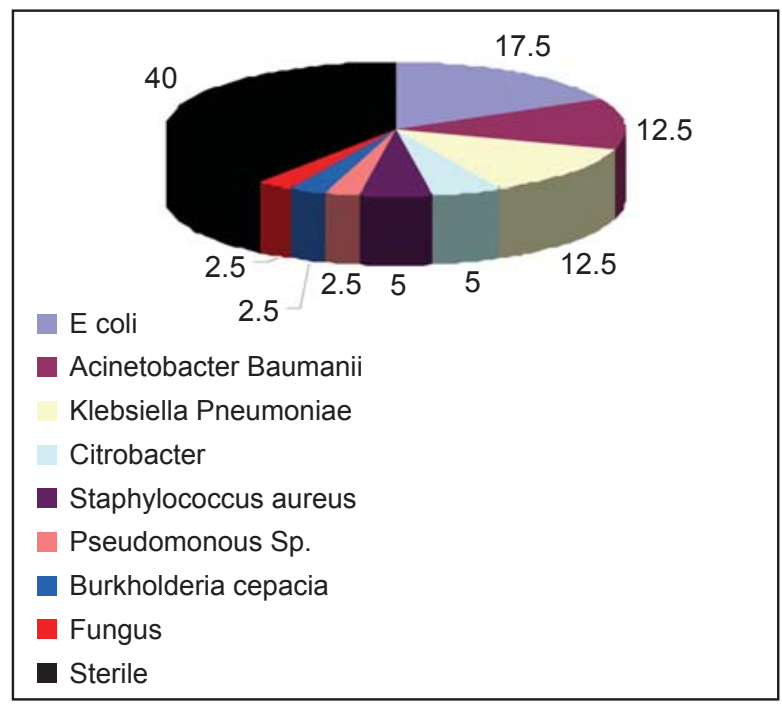

Fig. 2: Distribution of Cases According to Microbiological Growth on Blood Culture

Among the high risk factors, prematurity constituted the maximum number of cases i.e. $55 \%$ followed by meconium stained amniotic fluid in 35\%, premature rupture of membranes for $>24$ hours in $25 \%$, birth asphyxia in $25 \%$, prolonged or difficult labor in $22.5 \%$, febrile illness in mother in $20 \%$, more than three vaginal examination in $20 \%$ and other risk factor included home delivery, unsterile handling and cutting of cord, multiple gestation and faulty feeding practices in $25 \%$ of cases.

Table 2: Sensitivity, Specificity, PPV, NPV and Accuracy of Diagnostic Markers.

\begin{tabular}{|l|c|c|c|c|c|}
\hline \multicolumn{1}{|c|}{ Tests } & Sensitivity & Specificity & PPV & NPV & Accuracy \\
\hline CBC & $37.50 \%$ & $75.00 \%$ & $69.23 \%$ & $44.44 \%$ & $52.50 \%$ \\
\hline Micro-ESR & $50.00 \%$ & $62.50 \%$ & $66.67 \%$ & $45.45 \%$ & $55.00 \%$ \\
\hline IT Ratio & $62.50 \%$ & $56.25 \%$ & $68.18 \%$ & $50.00 \%$ & $60.00 \%$ \\
\hline CRP & $84.21 \%$ & $28.57 \%$ & $51.61 \%$ & $66.67 \%$ & $55.00 \%$ \\
\hline
\end{tabular}


In study group, CBC was abnormal in 26 cases (32.5\%), blood culture (BacTalert) was positive in 48 cases $(60 \%)$ and eight cases (10\%) had CBC abnormal with sterile blood culture (sensitivity $=37.50 \%$, specificity $=75.00 \%$ ).

CRP was reactive in 62 cases (77.5\%), blood culture was positive in 48 cases (60\%) and 30 cases (37.5\%) had CRP reactive with sterile blood culture. MicroESR was abnormal in 36 cases (45\%), blood culture was positive in 48 cases (60\%) and 12 cases (15\%) had Micro ESR abnormal with sterile blood culture. IT Ratio was abnormal in 44 cases (55\%), Blood culture was positive in 48 cases $(60 \%)$ and 14 cases $(17.5 \%)$ had IT Ratio abnormal with sterile blood culture.

\section{Discussion}

Neonatal sepsis is mainly a bacterial infection in the blood which is caused by bacterial, fungal, parasitic or viral pathogens. It is a condition found in infants less than one month old and a potentially serious infection that requires NICU care.

Our study revealed that males (70\%) were reported to be more likely than females to develop septicemia. Faridi and Gupta $(1992)^{3}$ also reported $66.67 \%$ males and $33.33 \%$ females out of 63 cases of neonatal septicemia.

In our study of 80 cases, 44 (55\%) were preterms while there were no preterm case in the control group. This showed increased risk of neonatal sepsis in premature newborns. This was similar to the observation made by Washburn and Medeasis ${ }^{4}$ where $53.97 \%$ preterm and $46.03 \%$ full term neonates.

We observed that prematurity and LBW were leading high risk factors for sepsis in 55\% of the newborns, other risk factors were meconium stained amniotic fluid (35\%) premature rupture of membrane for $>24$ hours (25\%), birth asphyxia (25\%), prolonged or difficult labour (22.5\%), febrile illness in the mother (20\%), >3 per-vaginal examination (20\%) and $25 \%$ others like, multiple gestation, home delivery, unsterile cord handling and cutting technique, faulty feeding practices, nursing in unhygienic environment, etc.

The most important risk factors for neonatal sepsis were prematurity and low birth weight as reported by Jain and Maheshwari et $\mathrm{al}^{5}$ in their study.

Blood culture is the gold standard method to diagnose septicemia. In our study, blood culture (BacTalert) had shown growth in 48 cases i.e.60\%
(46 bacterial and two fungal ) in the study group, while blood culture (BacTalert) was positive in two case $(2.5 \%)$ in the control group. The most common organism grown on blood culture was E.Coli (17.5\%). Jain and Maheshwari et $\mathrm{al}^{5}$ analyzed the signs and symptoms of neonatal sepsis in 106 neonates with suspected sepsis out of which 30 were culture positive. The most common organism was E. coli which is similar to this study.

Neonatal sepsis data from most of the medical centers in the US shows that $E$. coli and group $B$ streptococcus accounted for $50-70 \%$ of the cases. Mirf et $\mathrm{al}^{6}$ in their study of 50 cases also showed similar results. McCraken ${ }^{7}$, Faridi and Gupta ${ }^{3}$, Kumar GD et al ${ }^{8}$ have also reported that gram negative septicemia is more common than gram positive septicemia

In this study $\mathrm{CBC}$ had low sensitivity (37.50\%) and high specificity $(75.00 \%)$. Chan and $\mathrm{Ho}^{9}$ revealed in their study that abnormal $\mathrm{CBC}$ had the lowest sensitivity and PPV while abnormal ANC had the lowest specificity and NPV among them.

In this study CRP was reported most sensitive (84.21\%) but low specific $(28.57 \%)$, Franz AR et al ${ }^{10}$ showed that there is generally a delay of up to 24 hours between onset of symptoms of infection and a rise in serum CRP. Sensitivity of the test at presentation is only $40 \%$ that is, $60 \%$ of subsequently proven sepsis episodes will have a normal initial CRP.

In our study IT ratio was reported NPV of $50.0 \%$. Ghosh et al $^{11}$ studied 103 high risk neonates having predisposing perinatal factors or clinical suspicion of sepsis and found that an abnormal immature to total neutrophil (IT) ratio were the most sensitive indicators in identifying neonates with sepsis showing high negative predictive value over $94 \%$.

In our study Micro-ESR has shown sensitivity of $50.0 \%$, specificity of 62.5 . K.K. Diwakar and Rosul G ${ }^{12}$ studied on 114 term neonates for early neonatal sepsis. The sensitivity and specificity of the 'revised' MicroESR was $62.5 \%$ and $60.9 \%$ respectively in diagnosing culture proven sepsis.

\section{Conclusion}

CRP was found most sensitive, while $C B C$ was found to be most specific marker in neonatal sepsis.

Acknowledgements: I am extremely grateful and highly indebted to my honorable Guide Dr. Nitin N. Kadam, Professor \& HOD, Department of Pediatrics, 
MGM Medical College and Hospital, Navi Mumbai, for his invaluable guidance, supervision to complete this study. I thank Mr. Dattatraya Parle for helping me in this study.

Funding: None

Conflict of Interest: This article is based on the final thesis (part of compulsory thesis submission) submitted to Department of Paediatrics MGM Medical College and Hospital, University of Mumbai.

Permission from IRB: Yes

\section{References}

1. Gotoff SP. Neonatal Sepsis and meningitis: in: Nelson textbook of Paediatrics. (15th Edition). Eds Behraman RE, Kleigman RM, Arbin AM. Philadelphia, WB saunders company 1996: 528537.

2. Agrawal R, Sarkar N, Deorary AK and Paul VK. Sepsis in newborn. Ind J Paediatr 2001;68:114347.

3. Faridi MMA, Gupta P, Bhargava SK. Chest radiograph in neonatal sepsis. Ind Ped 1972;29:871.

4. Washburn TC, Medearis DN Jr, Childs B. Sex difference in susceptibility to infection. Pediatrics 1965;35;57-64.
5. Jain NK, Jain VM, Maheshwari S. Clinical Profile of Neonatal Sepsis. Kathmandu Univ Med J 2003; 1: 117-120.

6. F. Mirf, S. Aman, S. Raza Khan. Neonatal Sepsis: a Review with a Study of 50 Cases. J Trop Pediatr 1987;33(3):131-135 doi:10.1093/tropej/33.3.131.

7. McCraken and Shinefield. Changes in the pattern of neonatal septicemia and meningitis. Am J Dis Child 1966; 112:33.

8. Kumhar GD, Ramachandran VG, Gupta P. Bacteriological analysis of blood culture isolates from neonates in a tertiary care hospital in India. $J$ Health Popul Nutr 2002;20(4):343-7.

9. Chan DK and Ho LY. Usefulness of C-reactive protein in the diagnosis of neonatal sepsis. Sing Med J 1997; 38(6): 252-5.

10. Franz $A R$, et al. Reduction of unnecessary antibiotic therapy in newborn infants using interleukin-8 and C-reactive protein as markers of bacterial infections. Pediatrics 1999; 104:447-53.

11. Ghosh S, Mittal M, Jaganathan G. Early diagnosis of neonatal sepsis using a hematological scoring system, Indian J Med Sci 2001; 55: 495-500.

12. Diwakar KK, Rosul G, "Revised Look at MicroErythrocyte Sedimentation Rate in Neonates". Indian Pediatr 1999;36:703-705. 